\title{
LA GUERRA FRÍA Y EL EMPIRISMO LÓGICO. NOTA SOBRE LAS TESIS DE GEORGE REISCH
}

\author{
RESEÑA DEL LIBRO: Reisch, George, Cómo la guerra fría transformó la filosofía \\ de la ciencia. Hacia las heladas laderas de la lógica. Buenos Aires, Argentina, \\ Universidad Nacional de Quilmes Editorial (2009)
}

\section{Diego López Rosende}

En el libro Cómo la Guerra Fría transformó la filosofía de la ciencia. Hacia las heladas laderas de la lógica (Reisch 2009), George Reisch narra y comenta el derrotero del empirismo lógico en las décadas anteriores y posteriores a la segunda guerra mundial y proporciona material que pretende apuntalar su hipótesis central acerca del papel decisivo que la Guerra Fría habría desempeñado en la transformación del mismo. Según Reisch, el anticomunismo y anticolectivismo de la postguerra, la desconfianza del estudio científico del hombre y las presiones políticas sobre la comunidad universitaria y sobre sus miembros, proporcionaron un ambiente hostil que determinó la desaparición de las reivindicaciones políticas y culturales originarias del movimiento. Se trata de una tesis contundente y clara aunque, como veremos, cuestionable. En efecto, creemos que varios de los argumentos de Reisch, así como su uso de la evidencia documental, deben ser rectitificados o complementados. El propósito de esta nota ha sido explicar por qué.

De acuerdo con la cronología del autor, el proceso de despolitización del empirismo lógico tuvo lugar rápidamente, aproximadamente entre 1949 y 1955. Durante estos años, Philipp Frank, quien llevaba la antorcha de Neurath tras la muerte de éste, perdió el liderazgo del movimiento, que fue cooptado por Feigl, Reichenbach y - de algún modo - Carnap. Según la explicación de Reisch, el proyecto de Frank descarriló debido a su carácter política y socialmente comprometido. El de los otros, por el contrario, medró en virtud de una profesión de fe formalista, aislada de cuestiones sociales y en sintonía con las concepciones sobre los valores que caracterizaron culturalmente la Guerra Fría. 
Se dio por estos años, según el autor, un giro en el empirismo lógico que se manifiesta en la clase de tópicos abordados - y sobre todo los evitados - en los escritos de sus miembros a partir de los años cincuenta y en el contenido específico de los mismos. En particular, este cambio se habría manifestado en la exclusión de temas como la "planificación" y las conexiones entre ciencia y política y en el énfasis en la doctrina no-cognitivista de los valores, que sustraía las cuestiones éticas - en la interpretación de Reisch - de la crítica científicamente informada (pp. 412, 413). Con respecto a esta última cuestión, un acontecimiento clave fue, según el autor, la publicación en 1951 de la obra The Rise of Scientific Philosophy de Hans Reichenbach (Reichenbach 1951), cuya concepción de los valores se habría convertido en la ortodoxia de los empiristas lógicos en el contexto de la Guerra Fría, al punto que quienes se oponían a la misma veían "su estatura y su influencia” reducidas (p. 451).

Como vemos, la explicación que ofrece Reisch de la transformación del empirismo lógico atribuye un peso decisivo a una tesis particular: la del carácter éticamente neutral de las aserciones científicas (y la consiguiente incapacidad de la ciencia para decidir por sí misma cuestiones valorativas). El cambio mismo que Reichenbach y Feigl habrían introducido en los años cincuenta es incluso equiparado con el énfasis en esta tesis (p. 451). Esto, seguramente, suscitará varias dudas. En primer lugar, la tesis en cuestión parece ser demasiado débil como para que su adhesión determine la ruptura, aunque sólo sea en el plano teórico, con cualquier "agenda sociopolítica" del empirismo lógico. En segundo lugar, la hipótesis del autor sobre el rol de la Guerra Fría lo obliga a sostener la existencia de cierto consenso sobre la tesis opuesta en los años treinta y cuarenta. En efecto, Reisch sostiene, por ejemplo, que tanto Feigl como Carnap habrían defendido, en años previos a la Guerra Fría, la capacidad de la ciencia empírica para decidir cuestiones valorativas (p. 426). Sin embargo, atribuye en otras ocasiones a Carnap, y de hecho a la mayoría de los empiristas lógicos, una tesis neutralista sobre la ética sin distinción de épocas (p. 447).

Reisch propende a resolver estos problemas mediante un procedimiento acaso no intencional pero manifiesto: atenuar el contenido apolítico de la posición neutralista cuando ésta es defendida por empiristas lógicos en los años treinta y acentuarlo cuando es formulada por Feigl y Reichenbach en los años cincuenta. Este procedimiento, lamentablemente, acaba por ofrecernos una caricatura de la posición de estos autores. Su atribución más original a la concepción de Reichenbach, sin duda, es nada menos 
que "la prominente concepción absolutista de los valores sociales y políticos sobre la que se basa el anticomunismo norteamericano" (p. 430). Esto sin duda sorprenderá a más de un lector, ya que la posición no cognitivista de la ética defendida por los empiristas lógicos ha sido tradicionalmente atacada por relativista o aun peor, nihilista. En su explicación de esta sorprendente interpretación, el autor sostiene que Reichenbach defendía una concepción axiomática de los valores éticos (p. 425), tratándolos “como axiomas que no estaban sujetos a discusión” (p. 430). Así queda explicada, entonces, la conexión de la teoría ética de Reichenbach con el absolutismo de la Guerra Fría: "Del mismo modo que sucediera en el tratamiento de Reichenbach de los compromisos éticos, la libertad constituía un axioma a ser dado por sentado y que no debía ser cuestionado o disputado" (p. 431). También se nos recuerda oportunamente que Reichenbach fue un "asesor e investigador pagado por la RAND" (p. 420); Hacia las heladas laderas del cinismo habría sido acaso un subtítulo más exacto para esta historia.

Hemos sugerido que la descripción del autor de la teoría de Reichenbach es injusta, y no es difícil explicar por qué. Esta teoría, en efecto, era en realidad netamente contraria al absolutismo moral, y fue Reichenbach mismo quien señaló el peligro de concebir las proposiciones éticas como absolutas: "Si una persona fue indoctrinada para creer que las reglas morales constituyen verdades absolutas, será inhibido en gran medida de abandonar dichas reglas" (Reichenbach 1951, p. 300). Cuando Reichenbach se refería a los “axiomas” éticos, no hacía más que reformular el núcleo de una posición acerca de la ética sobre la cual había, excepto ligeros matices, consensus omnium entre los positivistas lógicos. Por estos axiomas entendía Reichenbach las proposiciones valorativas que constituyen premisas indispensables (aunque no necesariamene únicas) para justificar (o revisar) una propuesta ética en un contexto determinado; pues, según Reichenbach, ninguna ponderación ética puede reivindicarse o refutarse solamente mediante consideraciones fácticas (o analíticas) como las que encontramos en las aserciones científicas. Nada de esto implica, por cierto, que estas proposiciones valorativas se conciban (o peor, deban concebirse) como "absolutas y fijas" (para usar las palabras de Reisch, p. 499). Dado que es una cuestión central en el libro, podemos citar a Reichenbach para aclararla:

El filósofo científico nunca negará que las ciencias sociales desempeñen un rol importante en las aplicaciones de las decisiones éticas. Y no intenta decir que los así llamados axiomas éticos son premisas invariables que valen para todos los tiempos y 
todas las condiciones. Incluso las premisas éticas generales pueden cambiar con el ambiente social, y cuando se las llama axiomas, este término significa sólo que para el contexto considerado no son cuestionadas (Reichenbach 1951, ibid.).

Paradójicamente, Reisch utiliza este mismo párrafo en su caracterización de la posición de Reichenbach (pp. 421-423), pero escoge sólo las últimas siete palabras: "para el contexto considerado, no son cuestionadas", y las interpreta como enunciando lo que el filósofo y el científico hacen, o deben hacer, con las valoraciones que predominan en su país. Así llega a construir lo que denomina "la concepción axiomática de Reichenbach”, que resulta ser una filosofía absolutista y acomodaticia pero, como vemos, ajena por completo al pensamiento de Reichenbach. Cuando Reisch sugiere que "las voliciones éticas (...) podrían, sin embargo, ser entendidas como contextualizadas, analizables y ya no tratadas como absolutas o fijas" (p. 450), no está enunciando, como cree, una alternativa neurathiana a la posición de Reichenbach, sino un corolario a esta misma posición. "La filosofía de la ciencia construyó su hogar en las heladas laderas de la lógica cuando adoptó una concepción de los valores como absolutos y aislados de la crítica científicamente informada", escribe Reisch (p. 432). Pero, por lo visto, ella nunca adoptó una concepción semejante.

Herbert Feigl es otro empirista lógico a quien Reisch retrata con los colores más oscuros. Según Reisch, el clásico libro Readings in the Philosophy of Science, coeditado por Feigl en 1953, es un símbolo del cambio producido en el empirismo lógico en los años cincuenta. La introducción a esta obra (escrita por May Brodbeck) complace a los censores macartistas, sugiere Reisch, por su delimitación higiénica entre la ética y la política, por un lado, y la ciencia y su filosofía, por el otro. El tono políticamente acomodaticio de Readings también se revelaría en la exclusión de aquellos trabajos de Frank que hablan sobre los valores y la ciencia y de los escritos más "unificadores" de Neurath y Morris.

Sin embargo, el manejo de la evidencia empírica que hace Reisch para resaltar la función ideológica de la compilación de Feigl es - como en muchas otras ocasiones notoriamente despreocupado. Por ejemplo, el ensayo del propio Feigl sobre la filosofía de la ciencia y el humanismo tiene un contenido que Reisch juzga - creemos que correctamente- tan "socialmente comprometido" como las propuestas de Frank y Neurath, y, en el mismo sentido, Reisch resuelve el problema señalando que el ensayo fue tomado de un volumen de American Quarterly del año 1949, un año antes de que 
comenzara la avanzada anticomunista sobre la vida académica. Esto, desde ya, explica a lo sumo que Feigl haya decidido publicarlo antes de 1950, pero no explica por qué decidió volverlo a publicar en 1953; y éste es (dados los supuestos de Reisch) el problema.

Hay otros datos manifiestamente relevantes para su análisis de esta publicación que Reisch también pasa por alto. Por ejemplo, como apéndice de Readings aparece un pequeño ensayo de Albert Einstein sobre los valores en la ciencia donde éste explica, entre otras cosas, que los axiomas éticos no gozan de un carácter privilegiado ni están menos sujetos a examen práctico que los axiomas científicos, mostrando de paso que la comparación de los juicios valorativos con axiomas no involucra ningún compromiso absolutista. Aun más, como afirma el propio Reisch (p. 192), Einstein era una de las figuras científicas más sospechadas y atacadas por los anticomunistas norteamericanos. Pero lo más interesante del volumen - en relación con la tesis de Reisch - es la persona a quien Feigl encomendó el capítulo sobre la metodología de la economía: el famoso economista marxista Oskar Lange, quien defendió en los años treinta la posibilidad de planificación económica eficiente en el debate sobre el cálculo económico socialista iniciado por Mises y Hayek. Lange, además, había oficiado como mediador entre Roosevelt y Stalin y fue por ello un protagonista político crucial justo en la época histórica más denostada por los macartistas. Peor aún, comentaba en el pulcro ensayo incluido en el volumen de Feigl que en las economías actuales el sistema de mercado no es un asignador eficiente de recursos que contribuya al bienestar general (Feigl y Brodbeck 1953, p. 754). Si los escritos de Neurath, Frank y Morris eran mínimamente "rosados" en el contexto del macartismo - Reisch cree que lo eran - el escrito de Lange en los Readings pintaba a Feigl del color rojo más chillón.

¿Transformó entonces la Guerra Fría el empirismo lógico? Responder esta cuestión en profundidad está fuera del alcance de esta nota. Intentaremos simplemente comentar algunas condiciones necesarias para su esclarecimiento que no están elucidadas de manera satisfactoria en el libro de Reisch. La primera concierne al a quo del cambio: el presunto carácter originariamente político de la filosofía de la ciencia iniciada por los empiristas lógicos. Reisch aduce para ello, principalmente, las credenciales socialistas de algunos de sus miembros y algunas insinuaciones del Manifiesto del Círculo de Viena, así como la recepción solidaria por ciertos círculos de izquierda en Estados Unidos. Hay, sin embargo, otras cuestiones y elementos de juicio 
relevantes - y no muy favorables a su tesis - que Reisch podría haber incorporado con provecho a su exposición.

En primer lugar, podemos mencionar las declaraciones al respecto que aparecen en la importante Autobiografía intelectual de Carnap, de donde Reisch mismo extrae varias citas. Podemos leer allí que, si bien es probable que varios miembros del Círculo compartieran una visión ilustrada y favorable a alguna forma de organización racional de la sociedad, en el Círculo no se hablaba sobre política, y que ellos separaban claramente -a excepción de Neurath- la actividad filosófica de la política (Carnap 1963, p. 23). Otro de los asistentes a las reuniones del Círculo de Viena, Carl Hempel, también manifestó alguna vez que en el Círculo había un acuerdo de "dejar la política en la puerta de entrada". Naturalmente, esto no significa que los empiristas lógicos supusieran que sus contribuciones carecían de conexiones importantes con la sociedad y la política. Podemos citar aquí un fragmento del escrito aparecido en The New York Times en 1938 firmado por Waldemar Kaempffert, pero muy probablemente redactado por Neurath mismo, que nos puede dar un indicio acerca de las verdaderas proyecciones políticas de la Enciclopedia para la Unidad de la Ciencia en los años treinta (cf. Reisch, p. 96 y Galison 1996 p. 38):

Los hombres que hacen esta enciclopedia no tienen ningún deseo de entrar en la arena política pero todo el deseo de influir en los líderes intelectuales y asegurar la libertad de la investigación científica en todas partes. Los enciclopedistas del siglo XVIII tuvieron su parte en la caída de la tiranía de su tiempo sin comprometerse en luchas políticas. Así ocurre con esta expresión de opinión empírica.

La segunda y tercera oración de este preciso fragmento, tanto o más difíciles de conciliar con la posición de Reisch que la primera y que recuerdan el párrafo final del Manifiesto del Círculo de Viena (en especial las palabras "No todo adherente individual a la concepción científica del mundo será un luchador" (Hahn, Neurath y Carnap 2002), p. 123), son inexplicablemente omitidas en su cita del mismo texto; pero incluso el posicionamiento de los nuevos enciclopedistas por fuera de la arena política en la primera oración parece no haber sido descifrado por Reisch en su narrativa (p. 96). Notemos además que la función ilustrada de la filosofía de la ciencia que aquí se invoca, cuyo aporte fundamental para el cambio social es la promoción de la concepción científica, no es exclusiva del empirismo lógico de preguerra sino que pudo ser 
perfectamente reclamada por Feigl y Reichenbach en los años cincuenta - de hecho, lo fue.

En segundo lugar, cabe preguntar si la práctica del empirismo lógico durante los años treinta y cuarenta exhibe realmente un compromiso político definido que se haya desvanecido luego. Si se repasa el programa mismo esbozado en el Manifiesto, y las publicaciones de la Enciclopedia de ciencia unificada anteriores a la Guerra Fría, se advertirá que los aportes de los empiristas lógicos tuvieron también por aquella época un contenido abrumadoramente epistemológico y formal (sin contenidos éticos o políticos) que muestra una clara continuidad en lo que respecta al tipo de actividad intelectual que practicarían durante y luego de la Segunda Guerra. Los mismos datos proporcionados por Reisch (entre otros, las declaraciones políticamente prescindentes de las circulares de la Enciclopedia y la fuerte disputa entre Carnap y Neurath en los años treinta) no sugieren un viraje hacia el neutralismo en los años cincuenta, sino que apoyan más bien un relato inverso: pareciera que, al menos desde los años treinta, el Círculo tendió claramente hacia el profesionalismo neutral, con la resistencia parcial, pero cada vez más vehemente, de Neurath, cuya muerte impidió cualquier oportunidad de éxito; antes que de un coup conservador durante la Guerra Fría, parece haberse tratado de una rebelión abortada. Por ello, es sorprendente que Reisch no explique la interesante evidencia proporcionada a estos efectos por autores como Peter Galison (1996) que parece indicar que ya en los años treinta el empirismo lógico pierde en Estados Unidos cualquier conato político socialista, debido en parte - e irónicamente - a la influencia de uno de los héroes de la narrativa de Reisch, Charles Morris.

Otra cuestión importante concierne a la índole de las presiones que determinaron, en la hipótesis de Reisch, la despolitización del movimiento. No hay duda de que la presión del macartismo tuvo influencia sobre la vida académica e intelectual de Estados Unidos. Pero, ¿cuáles fueron los mecanismos constatables que inhibieron la supuesta impronta socialista del empirismo lógico, y a qué se debieron?

Podemos comentar aquí brevemente algunas de los datos específicos aducidos por Reisch. Un mecanismo inhibidor provino, según él, de los propios colegas filoempiristas de Philipp Frank, quienes habrían retaceado su apoyo al proyecto de unidad de la ciencia. Este retaceo habría consistido principalmente en la reticencia de estos autores a avalar en sus contribuciones intelectuales el rumbo "social" propuesto por Frank (pp. 358, 364). Ahora bien, de la evidencia aducida surge que los colegas de 
Frank respondieron generosamente a sus convocatorias de artículos y que contribuyeron con la única clase de escritos que estaban en condiciones de aportar: filosofía técnica de la ciencia y del lenguaje. Más aún, pareciera que el compromiso comunicacional e integrador de Neurath no fue puesto en práctica con mucha convicción por Frank y, más aún, que las presiones de la Academia de Ciencias que señala Reisch se debieron justamente a que este compromiso no estaba siendo honrado (pp. 359, 369).

Reisch también menciona el desprestigio intelectual que sufrió Frank en la comunidad académica. Se refiere en particular a una supuesta acusación pública de comunismo en una reseña de su libro Philosophy of Science (Frank 1957); pero el criterio hermenéutico del que se sirve para descifrar tal acusación, siquiera como posibilidad, en el texto que cita es francamente insondable; allí, además, se lo acusa de tomista, algo quizás preocupante, pero que más bien excluye cualquier imputación de comunismo. Por lo demás, el declive profesional de Frank, como admite Reisch, bien podría explicarse por su conducción errática y ambivalente del instituto de Unidad de la Ciencia (p. 369) y también, posiblemente, por su agotamiento intelectual. El compromiso de Frank con el operacionalismo, que ya había sido rotundamente criticado dentro y fuera del empirismo lógico, sin duda habría merecido una sección aparte al evaluar el status de Frank en la comunidad filosófica profesional.

Otro elemento de juicio aducido por Reisch son las investigaciones del FBI a Frank y a Carnap, que sin embargo no parecen haber sido motivadas por ninguna peculiaridad del proyecto propugnado por el primero, sino por las sospechas que era harto esperable que los macartistias tuvieran en relación con estos dos emigrados.

Mencionemos, por último, otro de los indicios que advierte Reisch: las sospechas de miembros de la fundación Rockefeller - que financió el instituto de Frank durante 6 años - de que podría haber algo "rosado", o al menos "extraño", en la Unidad de la Ciencia de los muchachos de Viena. Estas sospechas, empero, no parecen tener el peso potencial que les adjudica Reisch. El contexto en que las inserta (pp. 380-382) hará suponer a más de un lector que ellas están relacionadas con el cese del apoyo económico de la fundación; si lee la nota al pie y compara las fechas, sin embargo, advertirá que todas ellas fueron expresadas al menos un año antes de que se renovara la financiación por otros tres años (p. 368 y p. 381, nota 15). Por otra parte, la decisión posterior de la fundación de no renovar su financiación es explicable - como Reisch 
meritoriamente parece reconocer - por el hecho de que el instituto no alcanzara varios de sus objetivos explícitos (p. 368).

En conclusión: si la guerra fría despolitizó el empirismo lógico, necesitamos más y mejores argumentos para decidirlo.

\section{Blibliografía}

Carnap, R. (1963): Intellectual autobiography, en Schilpp (1963), pp. 3-81.

Feigl, H. y Brodbeck, M. (eds.) (1953): Readings in the Philosophy of Science, New York, Appleton-Century-Crofts.

Frank, P. (1957): Philosophy of science. The Link between Science and Philosophy, Englewood Cliffs, Prentice Hall.

Galison, P. (1996): Constructing Modernism: The cultural Location of the Aufbau, en Giere y Richardson 1996.

Giere, R. y Richardson, A. (eds.) (1996): Origins of Logical Empiricism, Minneapolis, University of Minnesota Press.

Hahn, H., Neurath, O. y Carnap, R. (2002 [1929]): La concepción científica del mundo: el círculo de Viena, REDES 9, número 18, pp. 103-149.

Reichenbach, H.: The Rise of Scientific Philosophy, Berkeley, University of California Press.

Reisch, G. (2009): Cómo la guerra fría transformó la filosofía de la ciencia. Hacia las heladas laderas de la lógica, Buenos Aires, Argentina, Universidad Nacional de Quilmes Editorial.

Schilpp, P. (1963): The Philosophy of Rudolf Carnap. La Salle, Illinois, Open Court. 